\title{
Output Tracking via Adaptive Backstepping Higher Order Integral Sliding Mode for Uncertain Nonlinear Systems
}

\author{
M. Pervaiz, ${ }^{1}$ Q. Khan, ${ }^{2}$ A. I. Bhatti, ${ }^{3}$ and S. A. Malik ${ }^{1}$ \\ ${ }^{1}$ Department of Electrical Engineering, COMSATS Institute of Information Technology (CIIT), Park Road, Chak Shahzad, \\ Islamabad 44000, Pakistan \\ ${ }^{2}$ Center for Advanced studies in Telecom, COMSATS (CIIT), Park Road, Chak Shahzad, Islamabad 44000, Pakistan \\ ${ }^{3}$ Department of Electronics Engineering, MAJU, Express Highway, Kahuta Road, Islamabad 44000, Pakistan
}

Correspondence should be addressed to M. Pervaiz; mahmood.pervaiz@gmail.com

Received 13 December 2013; Accepted 26 January 2014; Published 6 March 2014

Academic Editor: Jinde Cao

Copyright (C) 2014 M. Pervaiz et al. This is an open access article distributed under the Creative Commons Attribution License, which permits unrestricted use, distribution, and reproduction in any medium, provided the original work is properly cited.

The authors propose a new tracking control design strategy for uncertain non-linear systems which are convertible to SemiStrict Feedback Form (SSFF). The system in SSFF is first converted into new variables via existing adaptive backstepping control techniques. The control law is obtained by combining adaptive backstepping procedure and higher order integral sliding mode. The component of control law designed via backstepping is continuous which shows robustness against parametric uncertainties where as the discontinuous control component provides robustness against unmodeled dynamics and external disturbances. Since, this strategy relies on an integral manifold of the adaptively developed variables, therefore, the reaching phase is eliminated in this approach, which is an advantage in term of robustness. Furthermore, the parameters update law correctly provides the estimation of parameters which is again results in enhanced robustness of the strategy. The stability of proposed method is analysed theoretically and validated through a numerical example.

\section{Introduction}

The design process of nonlinear feedback control system is quite complex and challenging. Major concern is to design a controller that guaranties some specific behaviour of the system and is robust against unmodeled dynamics, parametric uncertainties, and external disturbances. There are no specific methods or set of analysis and design tools that can fit universally for wide range of situations and applications. However several tools are available which are generally applied to control nonlinear feedback system to achieve desired regulation or output tracking.

Sliding mode control (SMC) reported by $[1,2]$ is well known for its ability to compensate the external disturbance, unmodeled dynamics, and parametric uncertainties satisfying matching conditions. The classical SMC suffers from high frequency control switching the chattering phenomenon that severely damages the actuators and the system. Another limitation of this method is that it can only be applied to the systems of relative degree one. Relative degree is the relationship of output to the input of the system. The relative degree number is the count of derivatives of the output variable on which input variable appears. In the system which satisfies 01 relative degree condition, on taking first derivative of the output variable, input variable appears in the equation. Many physical systems, such as mechanical systems and satellite control system, do not satisfy 01 relative degree condition. The need of the relative degree one and chattering reduction/elimination was handled via different techniques (see, e.g., [3-5]).

A systematic design procedure that resolves the issue of relative degree is the backstepping algorithm [6] and it is suitable for feedback linearisable systems [7-9]. This is essentially a recursive design methodology in which a virtual control is designed at each step until an actual control law is realized. Conventional feedback linearisation method [3] has a drawback in the sense that this requires a precise model around some specific equilibrium points where some useful nonlinearities responsible for stability may disappear. However, the backstepping method avoids 
wasteful cancellations to achieve the global stability and to improve the performance.

Sira-Ramirez et al. [10] have combined the fundamental adaptive backstepping algorithm [11] with dynamically inputoutput linearization technique [7]. The blended method was extended in combination with the SMC, for those systems which can be transformed into triangular, Parametric Pure Feedback Form (PPFF) or Parametric Strict Feedback Form (PSFF) (see, e.g., [12-14]). Furthermore, some interesting SMC based techniques were put forwarded by, for example, [15-17] for nonlinear systems which are transferable into semistrict feedback form (SSFF) suffering from unmodeled and unmatched uncertainties. In this way, the benefit of SMC and adaptive backstepping were realised. An additional benefit is that the requirement of transformation into PPFF or PSFF and the justification of extra conditions on parameters and sufficient condition for the existence of sliding mode [14] were not needed. Recently, Khan et al. [18] reported strategy of dynamic control for MIMO uncertain, square, and minimum phase nonlinear system based on integral sliding mode technique. This method has synthesized dynamic sliding mode control $[19,20]$ and integral sliding mode strategies [1] into dynamically integral sliding mode control (DISMC) which provided the establishment of sliding mode without reaching phase. In this way the robustness against uncertainties is enhanced and performance is improved with considerable attenuation in the chattering across the integral manifold.

Fascinated by the strategy of DISMC, the authors have combined the adaptive backstepping and Higher Order Integral Sliding Mode Control (HOISMC) (see, e.g., [21, 22]) into a new technique named Adaptive Backstepping Higher Order Integral Sliding Mode Control (ABHOISMC); this will provide robustness from the initial time instant against parametric uncertainty along with considerable reduction in chattering. This newly proposed technique is developed for Single Input Single Output (SISO) nonlinear systems which are transferable into SSFF in the presence of some unknown parameters. The design process involves the subdivision of the control law into two parts, continuous control law which can be realized by applying adaptive backstepping method and discontinuous control law which is developed via an integral manifold. The parameter update law is also a sum of two terms. The first term emerges from the adaptive backstepping method and the second term is the contribution of the HOISMC. The remainder of the paper is managed as follows: the problem description/formulation is presented in Section 2, the development of the control law is explained in Section 3, an illustrative example is presented in Section 4, and the conclusions are drawn in Section 5.

\section{Preliminaries}

2.1. System Description. Consider a class of nonlinear systems which can be represented/transformed into the following SSFF [15]:

$$
\begin{gathered}
\dot{\zeta}_{i}=\zeta_{i+1}+\varphi_{i}^{T}\left(\zeta_{1}, \zeta_{2}, \ldots, \zeta_{i}\right) \theta+\eta_{i}(\zeta, \omega, t), \\
\dot{\zeta}_{n}=f(\zeta)+g(\zeta) u+\varphi_{n}^{T}(\zeta) \theta+\eta_{n}(\zeta, \omega, t), \\
y=\zeta_{1} ; \quad 1 \leq i \leq n-1,
\end{gathered}
$$

where $\zeta=\left[\zeta_{1}, \zeta_{2}, \ldots, \zeta_{n}\right]^{T}$ is the state vector, $u$ is the scalar control input, $y$ is the output of interest, $\varphi_{i}\left(\zeta_{1}, \zeta_{2}, \ldots, \zeta_{i}\right) \in$ $\mathfrak{R}^{p}, i=1, \ldots, n$ are known and sufficiently smooth functions, $f(\zeta)$ and $g(\zeta)$ are known multivariable functions, $\theta \in$ $R^{p}$ is the vector of unknown parameters, and $\eta_{i}(\zeta, \omega, t)$, $i=1, \ldots, n$, are the unknown nonlinear scalar functions including all the disturbances, whereas $\omega$ is an uncertain time-varying parameter.

It is required that the output $y$ of the system should follow a specific bounded desired continuous signal $y_{d}(t)$.

Assumption 1. It is assumed that the $n$th order time derivatives of $y_{d}$ exist and are bounded in nature. In addition, it is assumed that the scalar nonlinear functions $\eta_{i}(\zeta, \omega, t), i=$ $1, \ldots, n$. are bounded by known positive functions; that is,

$$
\begin{gathered}
h_{i}\left(\zeta_{1}, \zeta_{2}, \ldots, \zeta_{i}\right) \in \mathfrak{R}^{p} \\
\left|\eta_{i}(\zeta, \omega, t)\right| \leq h_{i}\left(\zeta_{1}, \zeta_{2}, \ldots, \zeta_{i}\right) .
\end{gathered}
$$

2.2. Adaptive Backstepping Design Approach Development. In this study, a generalised recursive approach analogous to that of [13] is presented for the tracking of a time varying signal $y_{d}(t)$. The step by step development is given as follows.

2.2.1. Step 1. Consider a new variable which represents the error between the actual output of the system and the desired output in the following form.

$$
z_{1}=\zeta_{1}-y_{d}(t) \text { and its derivative } \dot{z}_{1}=\dot{\zeta}_{1}-\dot{y}_{d}(t) \text { along (1) }
$$
becomes

$$
\dot{z}_{1}=\zeta_{2}+\varphi_{1}^{T}\left(\zeta_{1}\right) \theta+\eta_{1}(\zeta, \omega, t)-\dot{y}_{d}(t) .
$$

The expression in (3) may be written in an alternate form as follows:

$$
\dot{z}_{1}=\zeta_{2}+\omega_{1}(t)^{T} \hat{\theta}+\eta_{1}(\zeta, \omega, t)-\dot{y}_{d}(t)+\omega_{1}(t)^{T} \widetilde{\theta},
$$

where $\tilde{\theta}=\theta-\widehat{\theta}, \omega_{1}(t)=\varphi_{1}\left(\zeta_{1}\right)$, and $\widehat{\theta}$ is the estimated value of unknown parameters.

Now, a virtual control input for the stabilization of the first step is designed by considering the Lyapunov function as follows:

$$
V_{1}\left(z_{1}, \widehat{\theta}\right)=\frac{1}{2} z_{1}^{2}+\frac{1}{2} \widetilde{\theta}^{T} \Gamma^{-1} \widetilde{\theta},
$$

where $\Gamma$ is a positive definite matrix. The time derivative of $V_{1}$ along (3) takes the form

$$
\begin{gathered}
\dot{V}_{1}\left(z_{1,} \widehat{\theta}\right)=z_{1} \dot{z}_{1}-\widetilde{\theta}^{T} \Gamma^{-1} \dot{\hat{\theta}} \\
\dot{V}_{1}\left(z_{1}, \widehat{\theta}\right)=z_{1}\left(\zeta_{2}+\omega_{1}(t)^{T} \widehat{\theta}+\eta_{1}(\zeta, \omega, t)-\dot{y}_{d}(t)\right) \\
+\widetilde{\theta}^{T} \Gamma^{-1}\left(\Gamma \omega_{1} z_{1}-\dot{\hat{\theta}}\right) .
\end{gathered}
$$

Define the parameter update law of the fist stage $\dot{\hat{\theta}}=\tau_{1}=$ $\Gamma \omega_{1} z_{1}$ and the stabilizing control

$$
\alpha_{1}\left(\zeta_{1}, \widehat{\theta}, t\right)=-\omega_{1}(t)^{T} \widehat{\theta}-c_{1} z_{1}-\frac{h_{1}^{2} z_{1}}{h_{1}\left|z_{1}\right|+(\epsilon / n) e^{-a t}},
$$


where $c_{1}, a$, and $\epsilon$ are positive constants. The last term in $\alpha_{1}$ is a saturation function which avoids the chattering in the evaluation of the control input. Now, define a second new variable of the form

$$
z_{2}=\zeta_{2}-\alpha_{1}\left(\zeta_{1}, \widehat{\theta}, t\right)-\dot{y}_{d}(t)
$$

By using (9) and (8) in (7) and applying Assumption 1 in (2), one gets

$$
\dot{V}_{1}\left(z_{1}, \widehat{\theta}\right) \leq-c_{1} z_{1}^{2}+z_{1} z_{2}+\frac{\epsilon}{n} e^{-a t}+\widetilde{\theta}^{T} \Gamma^{-1}\left(\tau_{1}-\dot{\hat{\theta}}\right) .
$$

Consequently, one has

$$
\dot{z}_{1}=-c_{1} z_{1}+z_{2}+\omega_{1}(t)^{T} \widetilde{\theta}+\eta_{1}(\zeta, \omega, t)-\frac{h_{1}^{2} z_{1}}{h_{1}\left|z_{1}\right|+(\epsilon / n) e^{-a t}} .
$$

The second step is developed as follows.

2.2.2. Step 2. The time derivative of the new variable defined in (9) along (1) is given by

$$
\begin{gathered}
\dot{z}_{2}=\dot{\zeta}_{2}-\dot{\alpha}_{1}\left(\zeta_{1}, \widehat{\theta}, t\right)-\ddot{y}_{d}(t) \\
\dot{z}_{2}=\zeta_{3}+\varphi_{2}^{T}\left(\zeta_{1}, \zeta_{2}\right) \theta+\eta_{2}(\zeta, \omega, t)-\frac{\partial \alpha_{1}}{\partial \zeta_{1}} \dot{\zeta}_{1}-\frac{\partial \alpha_{1}}{\partial \widehat{\theta}} \\
-\frac{\partial \alpha_{1}}{\partial t}-\ddot{y}_{d}(t) .
\end{gathered}
$$

Now, define a Lyapunov function as follows.

$V_{2}=(1 / 2) z_{1}^{2}+(1 / 2) z_{2}^{2}+(1 / 2) \widetilde{\theta}^{T} \Gamma^{-1} \widetilde{\theta}$ and its time derivative along (11) and (12) becomes

$$
\begin{aligned}
\dot{V}_{2}\left(z_{1}, z_{2}, \widehat{\theta}\right)= & -c_{1} z_{1}^{2}+\frac{\epsilon}{n} e^{-a t} \\
& +z_{2}\left[z_{1}+\zeta_{3}+\omega_{2}^{T} \widehat{\theta}+\left(\eta_{2}-\frac{\partial \alpha_{1}}{\partial \zeta_{1}} \eta_{1}\right)\right. \\
& \left.\quad-\frac{\partial \alpha_{1}}{\partial \widehat{\theta}} \dot{\hat{\theta}}-\frac{\partial \alpha_{1}}{\partial \zeta_{1}} \zeta_{2}-\frac{\partial \alpha_{1}}{\partial t}-\ddot{y}_{d}(t)\right] \\
& +\widetilde{\theta}^{T} \Gamma^{-1}\left(\tau_{2}-\dot{\hat{\theta}}\right),
\end{aligned}
$$

where $\tau_{2}=\Gamma\left(\omega_{1} z_{1}+\omega_{2} z_{2}\right)$.

Now, define a third new variable of the form $z_{3}=\zeta_{3}-$ $\alpha_{2}\left(z_{1}, z_{2}, \hat{\theta}, t\right)-\ddot{y}_{d}(t)$. The selection of $\alpha_{2}\left(z_{1}, z_{2}, \widehat{\theta}, t\right)$ follows as

$$
\begin{aligned}
\alpha_{2}\left(z_{1}, z_{2}, \widehat{\theta}, t\right)= & -z_{1}-c_{2} z_{2}-\omega_{2}^{T} \widehat{\theta}-\frac{h_{2}^{2} z_{2}}{h_{2}\left|z_{2}\right|+(\epsilon / n) e^{-a t}} \\
& -\frac{h_{1}^{2} z_{2}}{h_{1}\left|z_{2}\left(\partial \alpha_{1} / \partial \zeta_{1}\right)\right|+(\epsilon / n) e^{-a t}}\left(\frac{\partial \alpha_{1}}{\partial \zeta_{1}}\right)^{2} \\
& +\frac{\partial \alpha_{1}}{\partial \zeta_{1}} \zeta_{2}+\frac{\partial \alpha_{1}}{\partial \widehat{\theta}} \tau_{2}+\frac{\partial \alpha_{1}}{\partial t}+\ddot{y}_{d}(t)
\end{aligned}
$$

and reduces (13) to the following form:

$$
\dot{V}_{2}\left(z_{1}, z_{2}, \hat{\theta}\right) \leq-c_{1} z_{1}^{2}-c_{2} z_{2}^{2}+z_{2} z_{3}+\frac{3 \epsilon}{n} e^{-a t},
$$

where $c_{2}$ is a positive constant, $\tau_{2}=\dot{\hat{\theta}}$, and

$$
\begin{aligned}
\dot{z}_{2}= & -z_{1}-c_{2} z_{2}+z_{3}+\omega_{2}^{T} \widetilde{\theta}+\left(\eta_{2}(\zeta, \omega, t)\right) \\
& -\frac{\partial \alpha_{1}}{\partial \zeta_{1}}-\left(\frac{h_{2}^{2}}{h_{2}\left|z_{2}\right|+(\epsilon / n) e^{-a t}}\right. \\
& \left.\quad-\frac{h_{1}^{2}}{h_{1}\left|z_{2}\left(\partial \alpha_{1} / \partial \zeta_{1}\right)\right|+(\epsilon / n) e^{-a t}}\left(\frac{\partial \alpha_{1}}{\partial \zeta_{1}}\right)^{2}\right) z_{2} \\
& +\frac{\partial \alpha_{1}}{\partial \hat{\theta}}\left(\tau_{2}-\dot{\hat{\theta}}\right) .
\end{aligned}
$$

Similarly, the recursive development in the new variables is in the sequel.

2.2.3. Step $k(1 \leq k \leq n-1)$. The variable $z_{k}$ is defined as $z_{k}=\zeta_{k}-\alpha_{k-1}-y_{d}^{(k-1)}$.

The time derivative of $z_{k}$ appears as follows:

$$
\begin{aligned}
\dot{z}_{k}= & \zeta_{k+1}+\varphi_{k}^{T}\left(\zeta_{1} \cdots \zeta_{k}\right) \hat{\theta}+\eta_{k}(\zeta, \omega, t)+\varphi_{k}^{T} \tilde{\theta} \\
& -\frac{\partial \alpha_{k-1}}{\partial t}-\frac{\partial \alpha_{k-1}}{\partial \widehat{\theta}}-\frac{\partial \alpha_{k-1}}{\partial \dot{\zeta}_{1}} \\
& -\frac{\partial \alpha_{k-1}}{\partial \zeta_{2}} \dot{\zeta}_{2} \cdots-\frac{\partial \alpha_{k-1}}{\partial \zeta_{k-1}} \dot{\zeta}_{k-1}-y_{d}^{(k)},
\end{aligned}
$$

where

$$
\dot{\zeta}_{k-1}=\zeta_{k}+\varphi^{T}\left(\zeta_{1}, \zeta_{2}, \ldots, \zeta_{k-1}\right) \theta+\eta_{k-1} .
$$

The uncertain time varying function in recursive procedure appears in the following form:

$$
\begin{gathered}
\omega_{k}=\varphi_{k}\left(\zeta_{1}, \ldots, \zeta_{k}\right)-\frac{\partial \alpha_{k-1}}{\partial \zeta_{1}} \varphi_{1}\left(\zeta_{1}\right) \\
-\frac{\partial \alpha_{k-1}}{\partial \zeta_{2}} \varphi_{2}\left(\zeta_{1}, \zeta_{2}\right) \cdots-\frac{\partial \alpha_{k-1}}{\partial \zeta_{k-1}} \varphi_{k-1}\left(\zeta_{1}, \ldots, \zeta_{k-1}\right), \\
\xi_{k}=\eta_{k}-\sum_{i=1}^{k-1} \frac{\partial \alpha_{k-1}}{\partial \zeta_{i}} \eta_{i},
\end{gathered}
$$

where $1 \leq k \leq n$.

The time derivative of the $z_{k}$ becomes

$$
\begin{gathered}
\dot{z}_{k}=\zeta_{k+1}+\omega_{k}^{T} \widehat{\theta}-\sum_{i=1}^{k-1} \frac{\partial \alpha_{k-1}}{\partial \zeta_{i}} \zeta_{i+1}-\frac{\partial \alpha_{k-1}}{\partial t} \\
-\frac{\partial \alpha_{k-1} \dot{\hat{\theta}}-\omega_{k}^{T} \widetilde{\theta}+\xi_{k}-y_{d}^{(k)}}{\partial \hat{\theta}}
\end{gathered}
$$


In addition, the next variable is defined according to the following formulation: $z_{k+1}=\zeta_{k+1}-\alpha_{k}-y_{d}^{(k)}$, where the virtual control input $\alpha_{k}$ appears in the subsequent form

$$
\begin{aligned}
\alpha_{k}(\zeta, \widehat{\theta}, t)= & -z_{k-1}-c_{k} z_{k}-\omega_{k}^{T} \widehat{\theta}+\sum_{i=1}^{k-1} \frac{\partial \alpha_{k-1}}{\partial \zeta_{i}} \zeta_{i+1} \\
& +\frac{\partial \alpha_{k-1}}{\partial t}-\xi_{k} z_{k}+\frac{\partial \alpha_{k-1}}{\partial \hat{\theta}} \tau_{k} \\
& -\left(\sum_{i=1}^{k-2} z_{i+1} \frac{\partial \alpha_{k-1}}{\partial \hat{\theta}}\right) \Gamma \omega_{k},
\end{aligned}
$$

where $c_{k}$ are positive constants. Now, substituting the previous available data, the $\dot{z}_{k}$ carry the following form:

$$
\begin{aligned}
\dot{z}_{k}= & -z_{k-1}-c_{k} z_{k}+z_{k+1}+\omega_{k}^{T} \widetilde{\theta} \\
& +\xi_{k}-\delta_{k} z_{k}-\frac{\partial \alpha_{k-1}}{\partial \widehat{\theta}}\left(\dot{\hat{\theta}}-\tau_{k}\right) \\
& +\left(\sum_{i=1}^{k-2} z_{i+1} \frac{\partial \alpha_{i}}{\partial \widehat{\theta}}\right) \Gamma \omega_{k},
\end{aligned}
$$

where $\delta_{k}$ is defined by

$$
\begin{aligned}
\delta_{k}= & \frac{h_{k}^{2}}{h_{k}\left|z_{k}\right|+(\epsilon / n) e^{-a t}} \\
& +\sum_{i=1}^{k-1}\left(\frac{\partial \alpha_{k-1}}{\partial \zeta_{i}}\right)^{2} \frac{h_{1}^{2}}{h_{i}\left|z_{k}\left(\partial \alpha_{k-1} / \partial \zeta_{i}\right)\right|+(\epsilon / n) e^{-a t}} .
\end{aligned}
$$

Now, the time derivative of a Lyapunov candidate function

$$
V_{k}\left(\zeta_{1}, \ldots, \zeta_{k}, \hat{\theta}\right)=V_{k-1}+\frac{1}{2} z_{k}^{2}
$$

takes the form

$$
\begin{aligned}
\dot{V}_{k} \leq & -\sum_{i=1}^{k} c_{i} z_{i}^{2}+z_{k} z_{k+1}+\frac{k(k+1) \epsilon}{2 n} e^{a t} \\
& +\widetilde{\theta}^{T} \Gamma^{-1}\left(\tau_{k}-\dot{\hat{\theta}}\right)+\left(\sum_{i=1}^{k-2} z_{i+1} \frac{\partial \alpha_{i}}{\partial \widehat{\theta}}\right) \\
& \times\left(\tau_{k}-\dot{\hat{\theta}}\right)
\end{aligned}
$$

where $\tau_{k}=\tau_{k-1}+\Gamma \omega_{k} z_{k}=\Gamma \sum_{i=1}^{k} \omega_{i} z_{i}$

2.2.4. Step $n$. Finally, define a variable $z_{n}$ as follows: $z_{n}=$ $\zeta_{n}-\alpha_{n-1}-y_{d}^{(n)}$, where the virtual control input $\alpha_{n-1}$ can be obtained by putting $k=n-1$ in (21). Thus, the final expression of $\dot{z}_{n}$ becomes

$$
\begin{aligned}
\dot{z}_{n}= & f(\zeta)+g(\zeta) u+\omega_{n}^{T}(\zeta, t) \hat{\theta}-\sum_{i=1}^{n-1} \frac{\partial \alpha_{n-1}}{\partial \zeta_{i}} \zeta_{i+1} \\
& -\frac{\partial \alpha_{n-1} \dot{\hat{\theta}}}{\partial \hat{\theta}}-\frac{\partial \alpha_{n-1}}{\partial t}+\omega_{n}^{T}(\zeta, t) \tilde{\theta}+\xi_{n}-y_{d}^{(n)},
\end{aligned}
$$

where the terms $\omega_{n}(\zeta, \widehat{\theta})$ and $\xi_{n}$ can be obtained from (19) by substituting $k=n$.

Note that, $z_{n}$ is the variable whose time derivative bears explicitly the actual control input $u$. In the next section, the control input is developed.

\section{Control Design via Higher Integral Sliding Mode}

In this study, which is the main contribution, a control design for the nonlinear systems convertible into SSFF is considered. The design is carried out by making use of first order and second order adaptive backstepping integral sliding mode control which are discussed in detail in forthcoming subsections.

3.1. Control Design via First Integral Sliding Mode. The control design for the systems which can be converted in new variables $\left[z_{1}, z_{2}, \ldots, z_{n}\right]^{T}$ is the study of this section. The authors have developed an integral sliding mode control with adaptive backstepping techniques. The control law appears as sum of a continuous and discontinuous component which may take the following mathematical form:

$$
u=u_{0}+u_{1}
$$

The main advantage of this technique is that sliding mode is enforced from the very beginning which enhances robustness against uncertainties. In addition, the system operates in sliding mode under the action of the continuous control component $u_{0} \in \mathfrak{R}$ [18] which is quite robust in this development because it is designed via the adaptive backstepping technique. The discontinuous component $u_{1} \in \mathfrak{R}$ comes into action, when the system reaches the vicinity of the sliding manifold. In addition, the parameter updates law is formulated as

$$
\dot{\hat{\theta}}=\dot{\hat{\theta}}_{0}+\dot{\hat{\theta}}_{1}
$$

where the first term comes from adaptive backstepping, while the second term comes from the sliding mode approach. In the subsequent study, the development is presented.

3.1.1. Design of the Adaptive Backstepping Controller $u_{0}$. The development of the continuous control component is presented in the form of the following proposition.

Proposition 2. Consider that the nonlinear system with new variables $\left[z_{1}, z_{2}, \ldots, z_{n}\right]$ exists, if a control law is chosen in the following form:

$$
\begin{aligned}
u=\frac{1}{g(\zeta)}[ & -z_{n-1}-c_{n} z_{n}-f(\zeta)-\omega_{n}^{T} \hat{\theta} \\
& +\sum_{i=1}^{n-1} \frac{\partial \alpha_{n-1}}{\partial \zeta_{i}} \zeta_{i+1}+\frac{\partial \alpha_{n-1}}{\partial \widehat{\theta}} \tau_{n}+\frac{\partial \alpha_{n-1}}{\partial t} \\
& \left.-\left(\sum_{i=1}^{n-2} z_{i+1} \frac{\partial \alpha_{i}}{\partial \widehat{\theta}}\right) \Gamma \omega_{n}+y_{d}^{(n)}-\delta_{n} z_{n}\right]
\end{aligned}
$$

with parameter update law

$$
\dot{\hat{\theta}}=\tau_{n}=\tau_{n-1}+\Gamma \omega_{n}^{T} z_{n} ;
$$

then the energy in this newly transformed system decays to zero asymptotically. 
Proof. Consider a Lyapunov candidate function of the form

$$
V_{n}=V_{n-1}+\frac{1}{2} z_{n}^{2}
$$

Calculating the time derivative of $V_{n}$ along (26) and then inserting the value of the control input $u$ from (29) and parameter update law from (30), one has

$$
\dot{V}_{n} \leq-\sum_{i=1}^{n} c_{i} z_{i}^{2}+\frac{(n+1) \epsilon}{2} e^{-a t} .
$$

Expression in (32) indicates that $V \rightarrow 0$ asymptotically (analogous to that of [17]), which ensures that the continuous control component is responsible for steering the actual system output to the desired output asymptotically.

Note that the update law mentioned in (30) is the first components of (28). In the forthcoming subsection, the design of the discontinuous term via integral sliding mode is presented.

3.1.2. The Design of the Discontinuous Component $u_{1} \in \mathfrak{R}$ of (27). In [17] a conventional sliding surface is taken for conventional adaptive sliding mode which is by definition a Hurwitz polynomial of the states. However, in the present development, an integral manifold is designed which results in reaching phase free sliding mode. The integral manifold under study is defined as follows:

$$
\sigma(z)=\sigma_{0}(z)+\varrho,
$$

where $\sigma_{0}(z)$ is the sliding manifold which usually appears as a linear combination of the states; that is, $\sigma_{0}(z)=\sum_{i=1}^{n} k_{i} z_{i}$, where $k_{i}>0, i=1, \ldots, n-1$ with $k_{n}=1$ are the designer parameters which are chosen according to the performance of the system. The second term on the right-hand side, that is, $\varrho$, is the integral term which always contains the nominal dynamics of the system. The design of $\varrho$ is presented in the following theorem.

Theorem 3. Consider the transformed system with the state vector $z=\left[z_{1}, z_{2}, \ldots, z_{n}\right]^{T}$. If the integral manifold is defined according to (33), the integral dynamics is chosen according to the following equation:

$$
\begin{aligned}
\dot{\varrho}= & \left(-k_{1} z_{2}+k_{1} c_{1} z_{1}-k_{2} z_{3}+k_{2} c_{2} z_{2}+\cdots-k_{n-1} z_{n}\right. \\
& \left.+k_{n-1} c_{n-1} z_{n-1}\right)+\left(k_{2} z_{1}+\cdots+k_{n-1} z_{n-2}\right) \\
& +\sum_{i=1}^{n-1} \frac{\partial \alpha_{n-i}}{\partial \zeta_{i}} \zeta_{i+1}+\frac{\partial \alpha_{n-1}}{\partial t}+y_{d}^{(n)}-g(\zeta) u_{0} \\
& +\frac{\partial \alpha_{n-1}}{\partial \widehat{\theta}} \tau_{n-1}-\omega_{n}^{T}(\zeta, t) \hat{\theta},
\end{aligned}
$$

and the discontinuous control component is selected as follows:

$$
u_{1}=-k \operatorname{sign}(\sigma) \text {, }
$$

where $k$ is positive constant equal to another constant $\mathrm{Kg}(\zeta)^{-1}$. Then sliding mode can be enforced along the integral manifold asymptotically.
Proof. We consider the Lyapunov function of the form

$$
V_{2}=\frac{1}{2} \sigma^{2}+\frac{1}{2} \widetilde{\theta}^{T} \Gamma^{-1} \widetilde{\theta}
$$

The time derivative of the above Lyapunov function along (33) becomes

$$
\dot{V}_{2}=\sigma\left(k_{1} \dot{z}_{1}+k_{2} \dot{z}_{2} \cdots k_{k-1} \dot{z}_{k-1}+\dot{z}_{n}+\dot{\varrho}\right)-\widetilde{\theta}^{T} \Gamma^{-1} \dot{\hat{\theta}},
$$

substituting the values of $\dot{z}_{i}$ from (22) where $i=1,2, \ldots, n$; one has

$$
\begin{aligned}
& \dot{V}_{2}=\sigma\left[k _ { 1 } \left(-c_{1} z_{1}+z_{2}+\omega_{1}^{T} \tilde{\theta}+\eta_{1}(\zeta, \omega, t)\right.\right. \\
& \left.-\frac{h_{1}^{2} z_{1}}{h_{1}\left|z_{1}\right|+(\epsilon / n) e^{-a t}}\right) \\
& +k_{2}\left(-z_{1}-c_{2} z_{2}+z_{3}+\omega_{2}^{T} \tilde{\theta}\right. \\
& +\left(\eta_{2}(\zeta, \omega, t)-\frac{\partial \alpha_{1}}{\partial \zeta_{1}} \eta_{1}(\zeta \omega, t)\right) \\
& -\left(\frac{h_{2}^{2}}{h_{2}\left|z_{2}\right|+(\epsilon / n) e^{-a t}}\right. \\
& -\frac{h_{1}^{2}}{h_{1}\left|z_{2}\left(\partial \alpha_{1} / \partial \zeta_{1}\right)\right|+(\epsilon / n) e^{-a t}} \\
& \left.\times\left(\frac{\partial \alpha_{1}}{\partial \zeta_{1}}\right)^{2}\right) \\
& \left.\left.\times\left(\frac{\partial \alpha_{1}}{\partial \zeta_{1}}\right)^{2}\right) \times z_{2}+\frac{\partial \alpha_{1}}{\partial \widehat{\theta}}\left(\tau_{2}-\dot{\hat{\theta}}\right)\right) \\
& \vdots \\
& +k_{n-1}\left(-z_{n-2}-c_{n-1} z_{n-1}\right. \\
& +\omega_{n-1}^{T} \widetilde{\theta}+\delta_{n-1} z_{n-1}+z_{n} \\
& -\frac{\partial \alpha_{n-2}}{\partial \widehat{\theta}}\left(\dot{\hat{\theta}}-\tau_{n-1}\right) \\
& \left.+\left(\sum_{i=1}^{k-3} z_{i+1} \frac{\partial \alpha_{i}}{\partial \widehat{\theta}}\right) \Gamma \omega_{n-1}\right) \\
& +f(\zeta)+g(\zeta)\left(u_{0}+u_{1}\right)+\omega_{n}^{T} \hat{\theta} \\
& -\sum_{i=1}^{n-1} \frac{\partial \alpha_{n-1}}{\partial \zeta_{i}} \zeta_{i+1}-\frac{\partial \alpha_{n-1}}{\partial \widehat{\theta}}-\frac{\partial \alpha_{n-1}}{\partial t} \\
& \left.+\omega_{n}^{T}(\zeta, t) \tilde{\theta}+\xi_{n}-y_{d}^{(n)}+\dot{\varrho}\right]-\widetilde{\theta}^{T} \Gamma^{-1} \dot{\hat{\theta}} .
\end{aligned}
$$

Now, inserting (34) in the above expression, one has

$$
\begin{aligned}
\dot{V}_{2}=\sigma & {\left[k_{1} \eta_{1}+k_{2}\left(\eta_{2}-\frac{\partial \alpha_{1}}{\partial \zeta_{1}} \eta_{1}(\zeta, \omega, t)\right)\right.} \\
& \left.+\cdots+k_{n-1} \xi_{n-1}+g(\zeta) u_{1}+\frac{\partial \alpha_{n-1}}{\partial \hat{\theta}}\left(\tau_{n}-\dot{\hat{\theta}}\right)\right] \\
+ & \widetilde{\theta}^{T} \Gamma^{-1}\left(\tau_{n}-\dot{\hat{\theta}}\right) .
\end{aligned}
$$


The terms $\tilde{\theta}$ in (39) will vanish by using parameter update law $\dot{\hat{\theta}}=\tau_{n}$, where $\tau_{n}=\Gamma \sigma\left(\sum_{i=1}^{n-1} \omega_{i}^{T} k_{i}+\omega_{n}\right)$.

Therefore, the above expression reduces to the following form:

$$
\dot{V}_{2}=\sigma\left[g(\zeta) u_{1}+k_{1} \eta_{1}+k_{2}\left(\eta_{2}-\frac{\partial \alpha_{1}}{\partial \zeta_{1} \eta_{1}}\right)+\cdots+\xi_{n-1}\right] .
$$

We denote $k_{1} \eta_{1}+k_{2}\left(\eta_{2}-\left(\partial \alpha_{1} / \partial \zeta_{1} \eta_{1}\right)\right)+\cdots=A$ and $\xi_{n-1}=B$, and, using (35), the expression in (40) takes the form

$$
\dot{V}_{2}=-k|\sigma|+|\sigma|[A+B]
$$

or

$$
\dot{V}_{2} \leq|\sigma|[-k+|A|+|B|] .
$$

Let $k>|A|+|B|$ and $-k+|A|+|B| \leq-\lambda$, where $\lambda>0$.

Thus,

$$
\dot{V}_{2} \leq-\lambda|\sigma|
$$

This proves that the sliding mode is enforced in finite time. However, the parameter behaves an asymptotic convergence. Thus, the overall system is asymptotically convergent.

Note that the parameter update law which appears before (40) is the second component of the parameter update law in (28). The algebraic sum of the two parameter update laws gives birth to the final expression of the parameters update law.

3.2. Second Order Adaptive Integral Sliding Mode Control Design (SOAISM). This subsection is dedicated to the study of second order adaptive integral sliding mode control design. Since higher order integral sliding modes (see, e.g., [21, 22]) are famous for their robustness from the very beginning with the use of an integral manifold, the real twisting and super twisting controller, reported in $[5,23]$, are utilized here to develop the discontinuous control component of the control law mentioned in (27). The mathematical expressions of the universal super twisting controller reported in [5] are given by

$$
u_{\text {dis }}=u_{1}+u_{2} \text {, }
$$

where

$$
u_{1}= \begin{cases}-\kappa\left|\sigma_{0}\right|^{l} \operatorname{sign}(\sigma), & |\sigma|>\sigma_{0}, \\ -\kappa|\sigma|^{l} \operatorname{sign}(\sigma), & |\sigma| \leq \sigma_{0},\end{cases}
$$

where $0<l \leq 0.5$ and

$$
u_{2}= \begin{cases}-u, & |u|>1 \\ -W \operatorname{sign}(\sigma), & |u| \leq 1\end{cases}
$$

The beauty of this controller is that it is of relative degree one and it does not need the derivatives of the sliding manifolds.
On the other hand, the real twisting controller needs the time derivative of the sliding manifold which will be recovered in this work via the uniform robust exact differentiator [24]. Assume that the error between the available signal $\sigma_{1}$ (which is the integral manifold in our case defined in (33)) and the estimated signal $\widehat{\sigma}_{1}$ is defined by $\widetilde{\sigma}=\widehat{\sigma}_{1}-\sigma_{1}$; then the first derivative can be estimated via the following algorithm:

$$
\dot{\hat{\sigma}}_{1}=\widehat{\sigma}_{2}-R_{1} \omega_{1}(\widetilde{\sigma}), \quad \dot{\hat{\sigma}}_{2}=-R_{2} \omega_{2}(\widetilde{\sigma}),
$$

where $R_{1}$ and $R_{2}$ are positive gains to be designed and

$$
\begin{gathered}
\omega_{1}(\widetilde{\sigma})=|\widetilde{\sigma}|^{0.5} \operatorname{sign}(\widetilde{\sigma})+\mu|\widetilde{\sigma}|^{3 / 2} \operatorname{sign}(\widetilde{\sigma}) \\
\omega_{2}(\widetilde{\sigma})=\frac{1}{2} \operatorname{sign}(\widetilde{\sigma})+2 \mu \widetilde{\sigma}+\frac{3}{2} \mu^{2}|\widetilde{\sigma}|^{2} \operatorname{sign}(\widetilde{\sigma}) .
\end{gathered}
$$

The output of $\dot{\hat{\sigma}}_{2}$, by integrating once, is $\widehat{\sigma}_{2}$ which is the first estimated derivative of $\sigma_{1}$ which is estimated very accurately. Since the real twisting controller, which appears as follows, will make use of the above estimated derivative:

$$
u_{\text {dis }}=-r_{1}\left(\widehat{\sigma}_{1}\right)-r_{2} \operatorname{sign}\left(\widehat{\sigma}_{2}\right) \quad r_{1}>r_{2}>0,
$$

the use of $u_{\text {dis }}$ refers to the second component of (27) which will enforce sliding mode against intersection of the manifolds $\sigma=\dot{\sigma}=0$ from the very beginning of the process. Consequently, the reaching phase will be eliminated and the chattering reduction will also occur across the switching manifold.

Note that the parameter update law in this case bears the first component of (28) because the higher order sliding manifold $\sigma=\dot{\sigma}=0$ contributes nothing to parameter update law. In the subsequent illustrative examples, the switching manifold and chattering reduction claim is verified. The parameter update law in this case becomes $\tau=\theta_{0}$ because $\sigma=0$ is justified from $t=0$.

\section{Illustrative Example}

This section is dedicated to verify the aforementioned claims. Therefore, consider a second order nonlinear system reported in [16] which is in the semistrict feedback form:

$$
\begin{gathered}
\dot{\zeta}_{1}=\zeta_{2}+\zeta_{1} \theta+A \zeta_{1}^{2} \cos \left(B \zeta_{1} \zeta_{2}\right), \\
\dot{\zeta}_{2}=u,
\end{gathered}
$$

where $A$ and $B$ are considered unknown, but it is known that $|A| \leq 2$ and $|B| \leq 3$. We also assume that

$$
\begin{gathered}
h_{1}=2 \zeta_{1}^{2}, \\
z_{1}=\zeta_{1}-y_{d}, \\
\alpha_{1}=-\zeta_{1} \hat{\theta}-c_{1} z_{1}+\frac{4 \zeta_{1}^{4} z_{1}}{h_{1}\left|z_{1}\right|+(\epsilon / 2) e^{(-a t)}}, \\
\omega_{1}=\zeta_{1}, \\
\omega_{2}=-\frac{\partial \alpha_{1}}{\partial \zeta_{1}} \zeta_{1}, \\
\dot{\hat{\theta}}_{0}=\Gamma\left(\omega_{1} z_{1}+\omega_{2} z_{2}\right), \\
4 \zeta_{1}^{4} \\
\delta_{2}=\frac{\partial \alpha_{1}}{2 \zeta_{1}^{2}\left|\left(\partial \alpha_{1} / \partial \zeta_{1}\right) z_{2}\right|+(\epsilon / 2) e^{(-a t)}}\left(\frac{2 \zeta_{1}}{2}\right.
\end{gathered}
$$




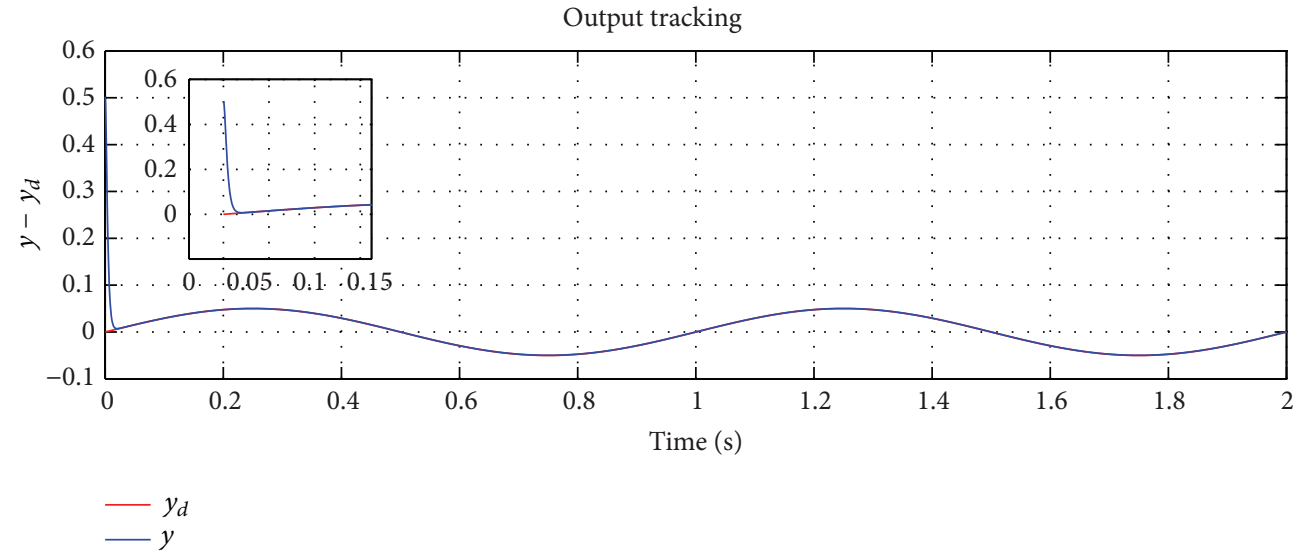

FIGURE 1: Output tracking of desired signal $y_{d}$.

Following the procedure of Section 2, the above system can be transformed into the new variables $z_{1}$ and $z_{2}$ as follows:

$$
\begin{aligned}
\dot{z}_{1}= & z_{2}-c_{1} z_{1}+2 \zeta_{1}^{2} \cos \left(3 \zeta_{1} \zeta_{2}\right) \\
& -\frac{4 \zeta_{1}^{4}}{2 \zeta_{1}^{2}\left|\left(\partial \alpha_{1} / \partial \zeta_{1}\right) z_{2}\right|+(\epsilon / 2) e^{(-a t)}}+\zeta \widetilde{\theta} \\
\dot{z}_{2}= & u-\frac{\partial \alpha_{1}}{\partial \zeta_{1}} \zeta_{2}+\omega_{2}^{T} \hat{\theta}+\omega_{2}^{T} \widetilde{\theta}-\frac{\partial \alpha_{1}}{\partial \zeta_{1}} 2 \zeta_{1}^{2} \cos \left(3 \zeta_{1} \zeta_{2}\right) \\
& -\frac{\partial \alpha_{1}}{\partial \hat{\theta}} \dot{\hat{\theta}}-\frac{\partial \alpha_{1}}{\partial t}-y_{d}^{(2)}
\end{aligned}
$$

Following the formulation of the continuous control law reported in (21), one gets

$$
\begin{aligned}
u_{0}= & -z_{1}-c_{2} z_{2}-\omega_{2}^{T} \hat{\theta}+\frac{\partial \alpha_{1}}{\partial \zeta_{1}} \zeta_{2}+\frac{\partial \alpha_{1}}{\partial \zeta_{1}} \omega_{1}^{T} \hat{\theta} \\
& +\frac{\partial \alpha_{1}}{\partial \widehat{\theta}} \tau_{2}+\frac{\partial \alpha_{1}}{\partial t}+y_{d}^{(2)}-\delta_{2} z_{2} .
\end{aligned}
$$

The discontinuous control component can be obtained by considering an integral manifold of the form

$$
\begin{aligned}
& \sigma=k_{1} z_{1}+z_{2}+\varrho, \\
& \dot{\sigma}=k_{1} \dot{z}_{1}+\dot{z}_{2}+\dot{\varrho} .
\end{aligned}
$$

For analysis, a Lyapunov function of the following form is considered:

$$
V_{2}=\frac{1}{2} \sigma^{2}+\frac{1}{2} \widetilde{\theta}^{T} \Gamma^{-1} \dot{\hat{\theta}}
$$

with the discontinuous control component as follows:

$$
u_{1}=-k \operatorname{sign}(\sigma) \text {. }
$$

The expression of the integral compensator dynamics is given by

$$
\begin{aligned}
\dot{\varrho}= & -k_{1} z_{2}+k_{1} c_{1} z_{1}+\frac{\partial \alpha_{1}}{\partial \zeta_{1}} \zeta_{2}+\omega_{2}^{T} \hat{\theta} \\
& +\frac{\partial \alpha_{1}}{\partial t}+y^{(2)}-u_{0}+\frac{\partial \alpha_{1}}{\partial \widehat{\theta}} \tau_{2},
\end{aligned}
$$

where the parameters update law in case of adaptive first order integral sliding mode is given by

$$
\dot{\hat{\theta}}_{1}=\Gamma \sigma\left(\omega_{1}^{T} k_{1}+\omega_{2}\right)
$$

The final parameter update law can then become

$$
\tau_{2}=\dot{\hat{\theta}}_{0}+\dot{\hat{\theta}}_{1}
$$

and the final expression of the control law becomes

$$
u=u_{0}+u_{1}
$$

Using the approach presented in the Theorem 3, one gets

$$
\dot{V}_{2} \leq-\lambda|\sigma|
$$

This expression shows that the sliding mode is enforced in finite time. However parameter convergence is asymptotic. Thus the overall system is asymptotically convergent.

The above example is simulated with the control law and parameter update laws defined above. The tracking of the $y_{d}=0.05 \sin (2 \pi t)$ is shown in Figure 1 which indicates that the time varying signals can be tracked very accurately in presence of uncertainties shown in Figure 7. The first order integral manifold displayed in Figure 3 ensures the sliding mode enforcement from the very beginning and, consequently, ensures robustness. The respective control input and parameter estimates are shown in Figures 4 and 2, respectively. Thus it is clear from these figures that the control input is appearing with reduced chattering.

The AHOISMC results of tracking are somewhat similar to that reported in Figure 1. Therefore, it is not displayed here. The clear advantage comes in the chattering reduction which is clear from Figures 3 and 6 comparison. That higher order sliding mode with adaptation provides almost chatter free control input. The sliding manifold convergence of this strategy is displayed in Figure 5. In addition, the phase trajectory of $\sigma=\dot{\sigma}=0$ is given in Figure 6 which, once again, shows the establishment of higher order sliding modes from the very beginning. This is a clear advantage of this proposed technique over the existing adaptive sliding modes 


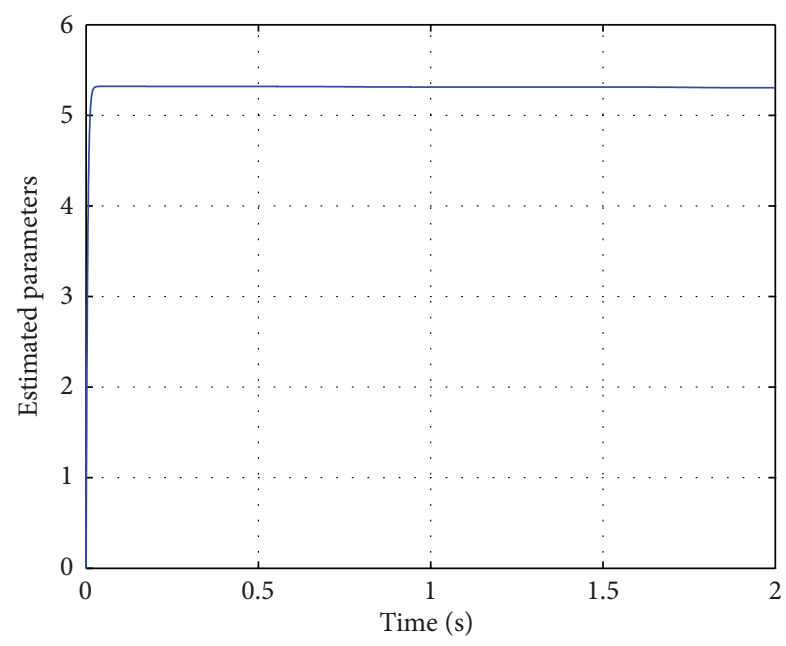

_ Estimated parameter

FIGURE 2: The estimated parameter.

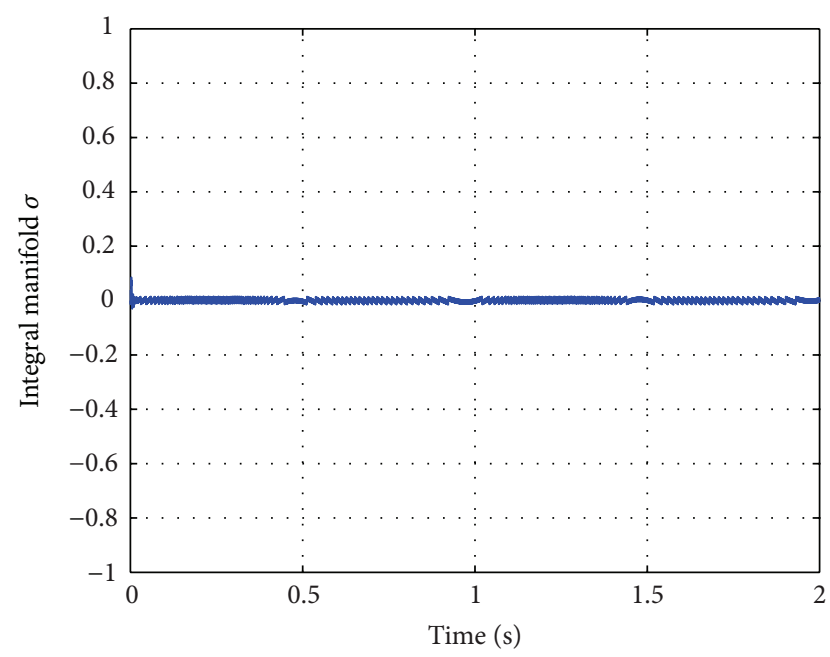

$\sigma$ convergence

FIGURE 3: Integral manifold $\sigma$ convergence.

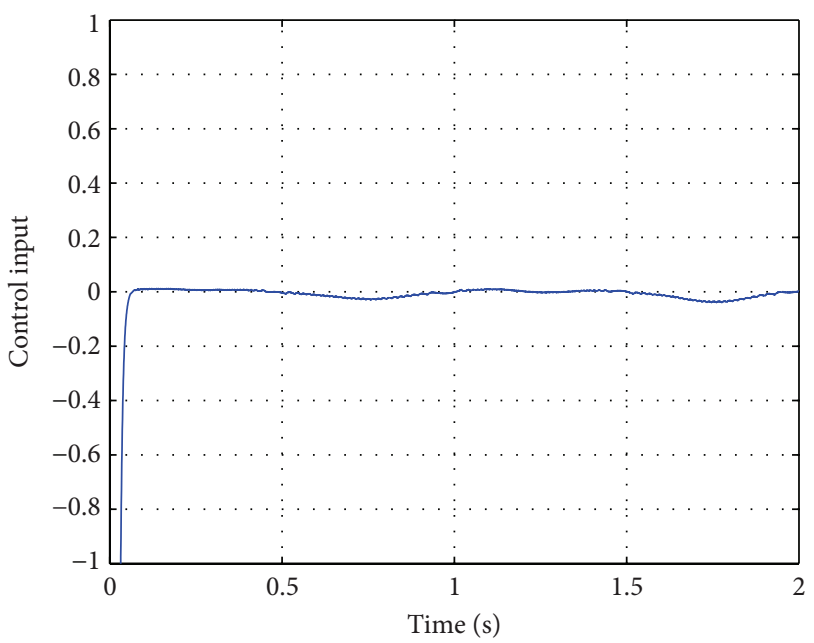

— Control input

FIGURE 4: The applied control input.

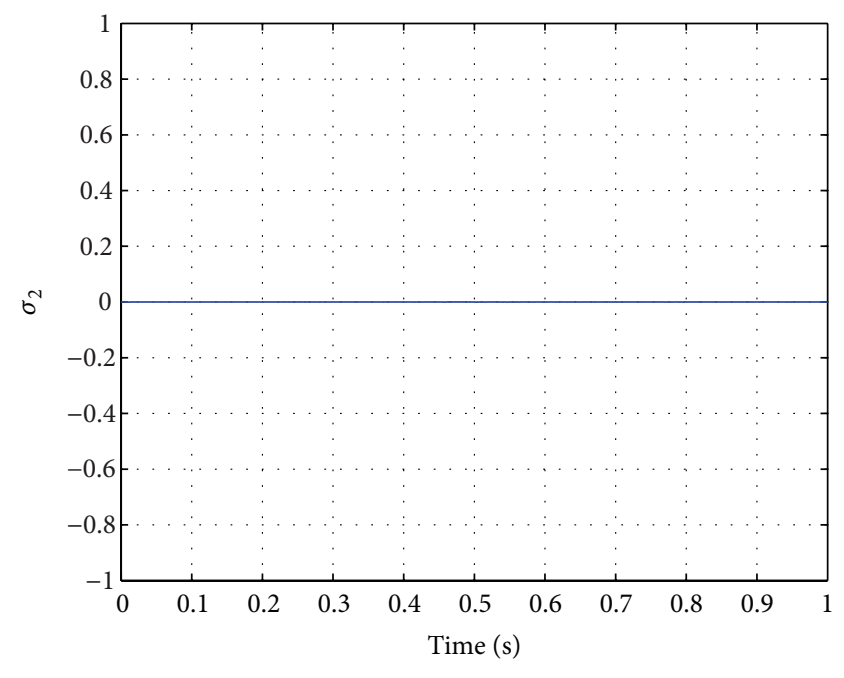

$-\sigma_{2}$

FIgURE 5: The $\dot{\sigma}$ convergence via the SOAISM.

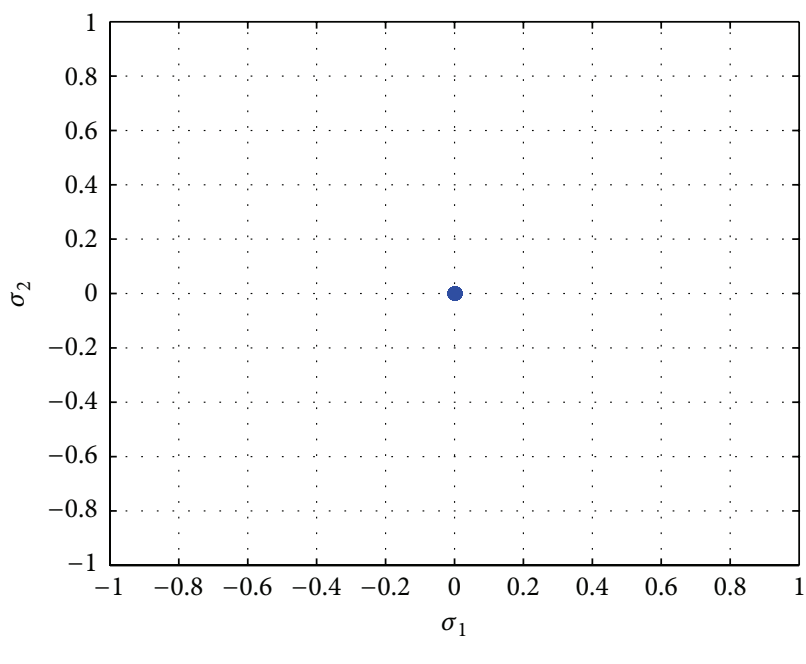

FIGURE 6: The manifold $\sigma_{1}=\sigma_{2}=0$ (phase portrait of $\sigma_{1}=\sigma_{2}=0$ ) for SOAISM.

techniques. The parameters used in simulation results are $\epsilon=1.997, a=0.7, k=30, k_{1}=1, c_{1}=200$, and $c_{2}=140$, $\lambda=0.1$.

From the aforementioned discussions of figures, it is verified that our new proposed techniques provide robustness from the very beginning via the integral manifold approach and the robustness against parametric variations is provided via adaptive backstepping. It is therefore claimed that the above development outshines the existing adaptive sliding mode techniques.

Comment. Proposed design technique is recursive; therefore it is costly in computation and complex due to complex mathematical derivations. Thus its computational cost is relatively higher than adaptive sliding mode. However beauty of this method is that its control action is global and robust. 


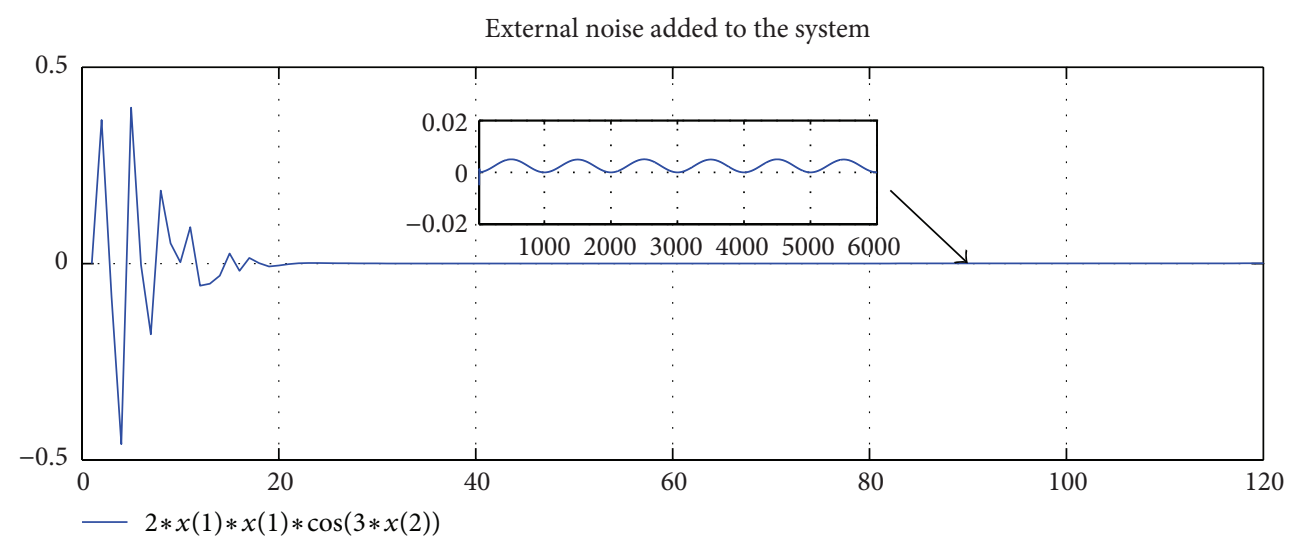

FIgURE 7: Output disturbance added.

\section{Conclusion}

The backstepping technique which is famous for its robust nature against parameter variation is utilized in combination with integral higher order sliding mode control strategy in order to enhance the robustness of the system from the very beginning of the process with considerable attenuation in chattering. The approach is relying on an adaptively developed new system. The integral manifold is designed in the new state variables. In other words, the manifold is adaptive in nature because of the adaptively developed states variable. The control law is designed via higher order integral sliding mode with adaptation which is capable of providing robustness against uncertainties caused by external/internal disturbances. The stability analysis is elaborated in terms of a proposition and a theorem. A numerical simulation result has verified the design approach.

\section{Conflict of Interests}

The authors declare that there is no conflict of interests regarding the publication of this paper.

\section{References}

[1] V. I. Utkin, J. Guldner, and J. Shi, Sliding Mode Control in Electromechanical Systems, Taylor and francsis, London, UK, 1999.

[2] C. Edwards and S. Spurgeon, Sliding Mode Control: Theory and Applications, Taylor and Francis, 1998.

[3] J. J. Slotine and W. Li, Applied Nonlinear Control, Prentice-Hall International, Taylor and Francis, 1991.

[4] S. V. Emelyanov, S. K. Korovin, and L. V. Levantovsky, "Higher order sliding regimes in the binary control systems," Soviet Physics, Doklady, vol. 31, no. 4, Article ID 291293, 1986.

[5] A. Levant, "Sliding order and sliding accuracy in sliding mode control," International Journal of Control, vol. 58, no. 6, pp. 12471263, 1993.

[6] M. Krstić, I. Kanellakopoulos, and P. V. Kokotović, "Adaptive nonlinear control without overparametrization," Systems \& Control Letters, vol. 19, no. 3, pp. 177-185, 1992.
[7] M. Fliess, "Nonlinear control theory and differential algebra," in Modelling and Adaptive Control, I. Byrnes and A. Khurzansky, Eds., vol. 105 of Lecture Notes in Control and Information Sciences, pp. 134-145, Springer, Berlin, Germany, 1989.

[8] I. A. Tall, "State and feedback linearizations of single-input control systems," Systems \& Control Letters, vol. 59, no. 7, pp. 429441, 2010.

[9] N. K. Mahdi, State and feedback linearization of single-input non-linear differential algebraic control systems [M.S. thesis], College of Science-University of Basrah, 2012.

[10] H. Sira-Ramirez, M. Rios-Bolivar, and A. S. I. Zinober, "Adaptive input-output linearization for PWM regulation of DC-toDC power converters," in Proceedings of the 1995 American Control Conference. Part 1, pp. 81-85, June 1995.

[11] M. Krstic, I. Kanellakopoulos, and P. V. Kokotovic, Nonlinear and Adaptive Control Design, Wiley, 1995.

[12] E. M. Rios-Bolívar and A. S. I. Zinober, "A symbolic computation toolbox for the design of dynamical adaptive nonlinear control," Applied Mathematics and Computer Science, vol. 8, no. 1, pp. 73-88, 1998.

[13] I. Kanellakopoulos, P. V. Kokotović, and A. S. Morse, "Systematic design of adaptive controllers for feedback linearizable systems," IEEE Transactions on Automatic Control, vol. 36, pp. 1241-1253, 1991.

[14] M. Rios-Bolivar and A. S. Zinober, "Dynamical adaptive backstepping control design via symbolic computation," in Proceedings of the 3rd European Control Conference, Brussels, Belgium, 1997.

[15] A. J. Koshkouei and A. S. I. Zinober, "Adaptive sliding backstepping control of nonlinear semi-strict feedback form systems," in Proceedings of the 7th IEEE Mediterranean Conference on Control and Automation, pp. 2376-2383, 1999.

[16] A. J. Koshkouei and A. S. I. Zinober, "Adaptive output tracking backstepping sliding mode control of nonlinear systems," in Proceedings of the 3rd IFAC Symposium Robust Control Design, vol. 1, pp. 167-172, 2000.

[17] A. J. Koshkouei, K. Burnham, and A. S. I. Zinober, "Dynamic siding mde cntrol for nnlinear sstems," IEE Proceedings of Control Theory and Applications, vol. 152, pp. 392-396, 2005.

[18] Q. Khan, A. I. Bhatti, S. Iqbal, and M. Iqbal, "Dynamic integral sliding mode for MIMO uncertain nonlinear systems," Inte- 
rnational Journal of Control, Automation and Systems, vol. 9, no. 1, pp. 151-160, 2011.

[19] X.-Y. Lu and S. K. Spurgeon, "Output feedback stabilization of SISO nonlinear systems via dynamic sliding modes," International Journal of Control, vol. 70, no. 5, pp. 735-759, 1998.

[20] X.-Y. Lu and S. K. Spurgeon, "Output feedback stabilization of MIMO non-linear systems via dynamic sliding mode," International Journal of Robust and Nonlinear Control, vol. 9, no. 5, pp. 275-305, 1999.

[21] S. Laghrouche, F. Plestan, and A. Glumineau, "Higher-order sliding mode control based on integral sliding mode," Automatica, vol. 43, no. 3, pp. 531-537, 2007.

[22] A. Levant and L. Alelishvili, "Integral high-order sliding modes," IEEE Transactions on Automatic Control, vol. 52, no. 7, pp. 1278-1282, 2007.

[23] A. Levant, "Universal (SISO) sliding-mode controllers with finite-time convergence," IEEE Transactions on Automatic Control, vol. 46, no. 9, pp. 1447-1451, 2001.

[24] E. Cruz-Zavala, J. A. Moreno, and L. M. Fridman, "Uniform robust exact differentiator," IEEE Transactions on Automatic Control, vol. 56, no. 11, pp. 2727-2733, 2011. 


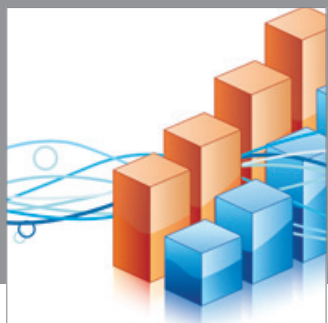

Advances in

Operations Research

mansans

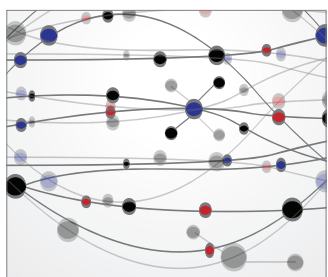

The Scientific World Journal
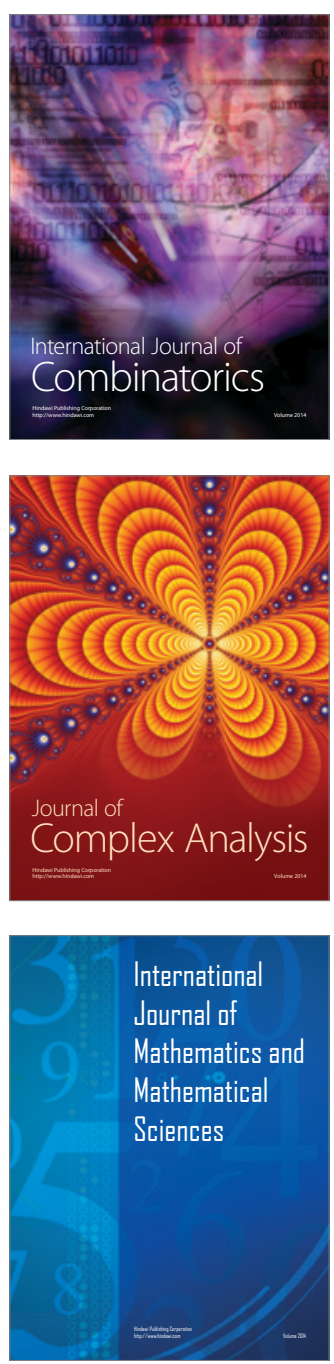
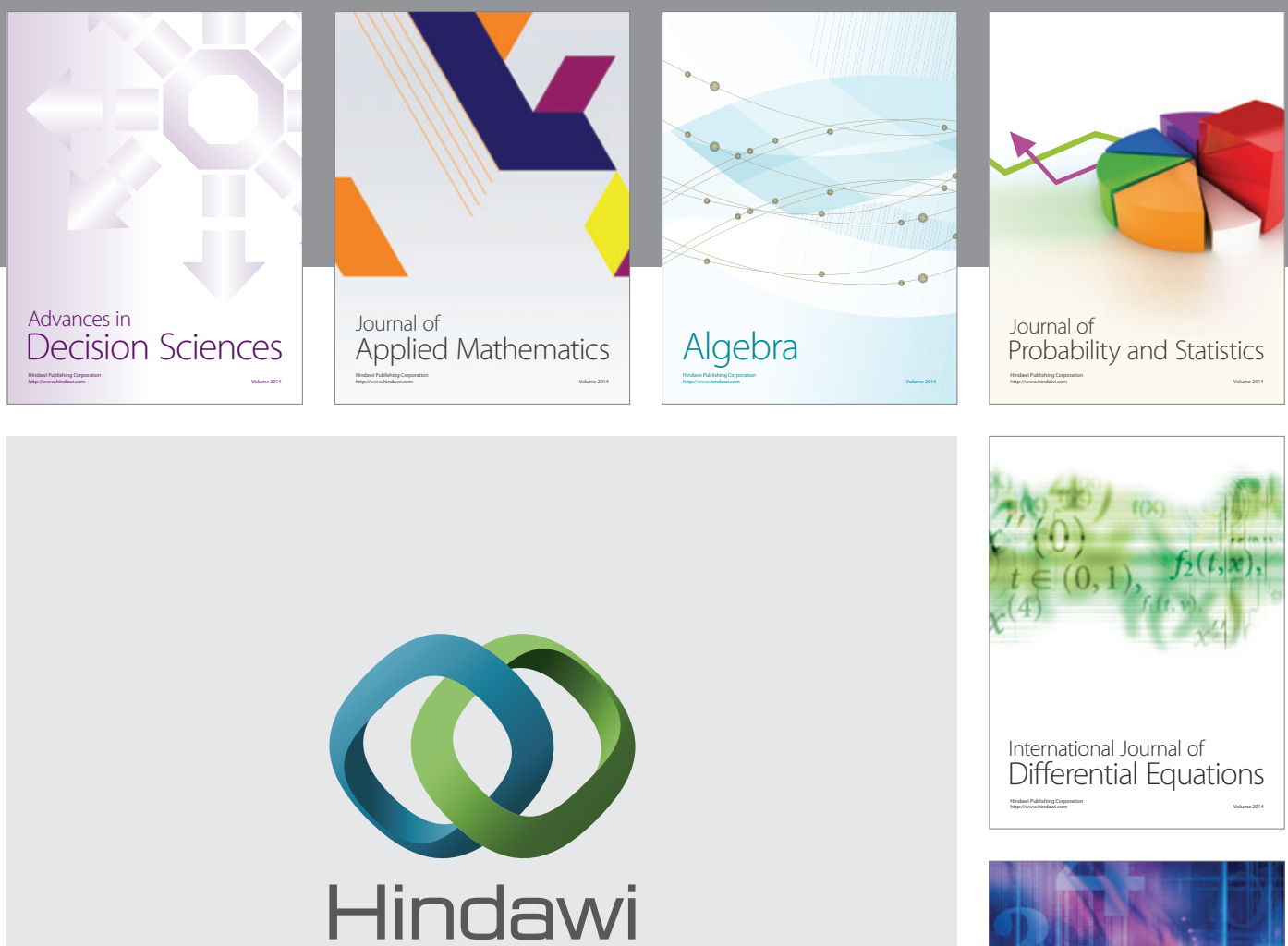

Submit your manuscripts at http://www.hindawi.com
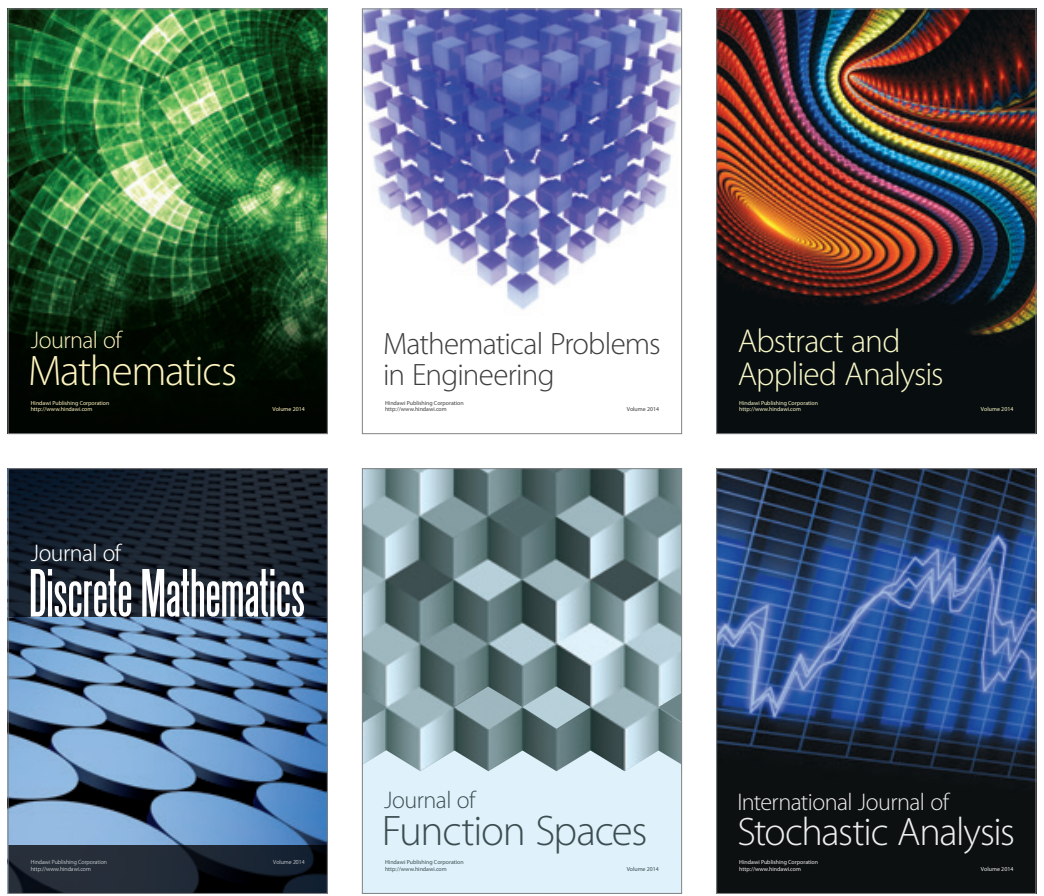

Journal of

Function Spaces

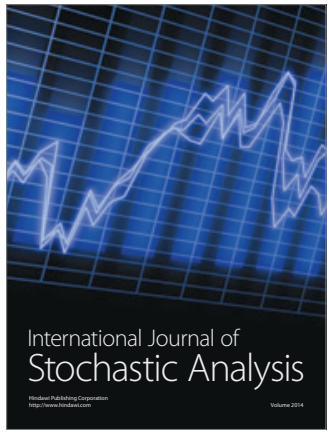

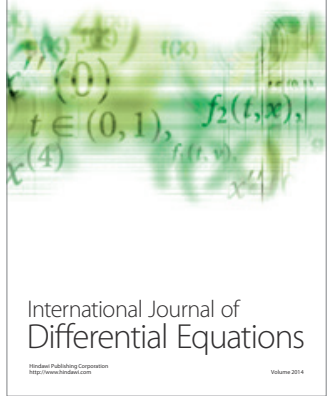
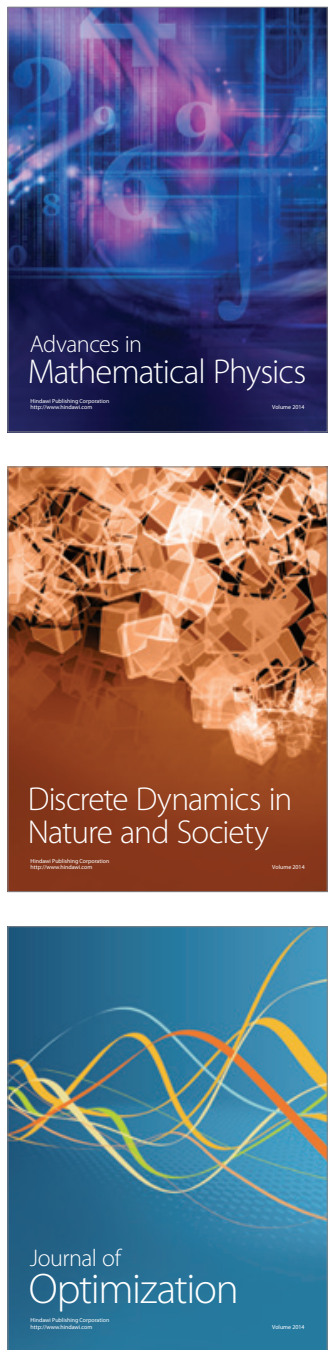